\title{
Effects of Processing Parameters on Functionally Graded Materials for Industrial Components Repair
}

\author{
André Alves Ferreira ${ }^{1,2 *}$, Ana Rosanete Reis ${ }^{1,2}$, João Manuel Cruz ${ }^{3}$ and Manuel Fernando Vieira' ${ }^{1,2 *}$ \\ ${ }^{1}$ Faculty of Engineering of the University of Porto, R. Dr. Roberto Frias, Portugal \\ ${ }^{2}$ LAETA/INEGI - Institute of Science and Innovation in Mechanical and Industrial Engineering, R. Dr. Roberto Frias, Portugal \\ ${ }^{3}$ SERMEC-Group, R. de Montezelo 540, Portugal
}

*Corresponding author: André Alves Ferreira and Manuel Fernando Vieira, Faculty of Engineering of the University of Porto, R. Dr. Roberto Frias, 4200-465 Porto, Portugal.
Received Date: August 23, 2021

Published Date: September 28, 2021

\begin{abstract}
The production of functionally graded materials (FGM) is an option for various industrial sectors and a solution for many engineering applications. FGMs are a class of materials that gradually change composition and structure, and whose properties vary throughout the material, depending on the spatial position in the material bulk structure. This article presents FGMs briefly as new materials for components repair using the direct laser deposition technique, emphasizing processing parameters and process optimization.
\end{abstract}

Keywords: Additive manufacturing; Functionally graded material; Direct laser deposition; Optimization

Abbreviations: FGM: Functionally Graded Materials; FGAM: Functionally Graded Additive Manufacturing; AM: Additive Manufacturing; SEM: Scanning Electron Microscopy; EDX: Energy Dispersive X-ray Spectroscopy; DLD: Direct Laser Deposition; CTE: Coefficient of Thermal Expansion

\section{Mini Review}

Functionally Graded Materials (FGMs) are a particular class of metal composites with a spatial variation of properties along a specific direction. By choosing FGMs, one can meet material requirements that are otherwise impossible to achieve. Among the FGMs advantages, the following stand out: (1) bonding of two complex and incompatible materials; (2) diminishing of the internal residual stresses; and (3) reduction of the crack driving force developed within the materials [1].

In recent years, Additive Manufacturing (AM) technologies evolved from producing conceptual prototypes to full-scale enduse components. The technological advancement of AM systems associated with the FGM approach led some authors to propose the concept of Functionally Graded Additive Manufacturing (FGAM) [2]. This layer-by-layer fabrication technique involves gradationally varying the material organization within a component to meet an intended function (Figure 1).

Additive Manufacturing (AM) is an important approach to build Metallic FGMs [3]. These consist of two (or more) different metals, or their alloys, combined in a melt pool generated by a heat source. In FGMs produced by Direct Laser Deposition (DLD), an AM process widely used in metallic materials, a laser is used as the energy input. DLD has the advantage of locally synthesizing alloys by mixing different powders with the desired composition. By gradually varying the mixing at various locations, parts with graded material properties can be generated [4]. 


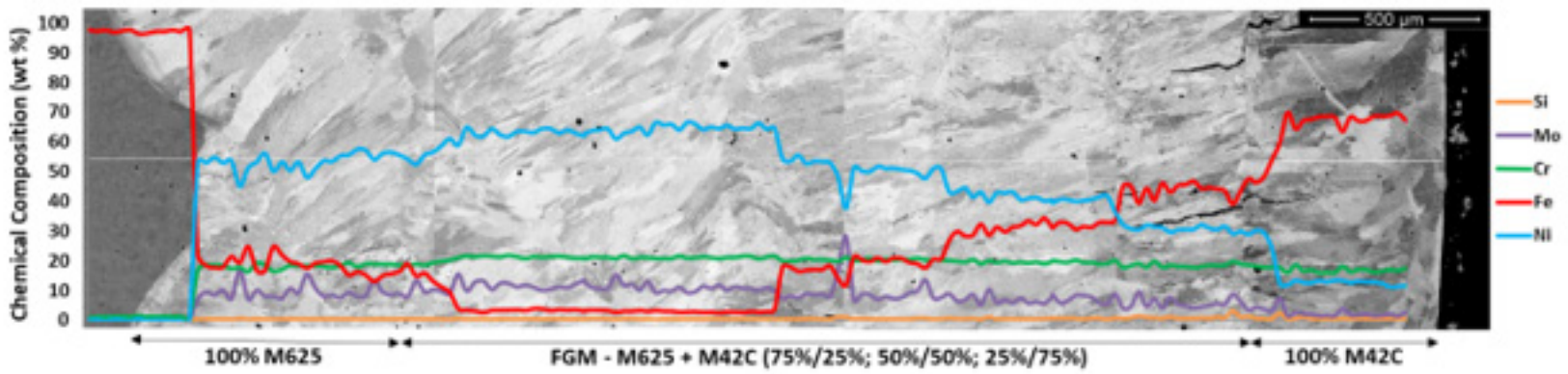

Figure 1: SEM image of microstructural evolution along the longitudinal section of an FGM. Superimposed on the image is the linear chemical analysis obtained by EDX. M625 - Nickel-based superalloy (Inconel 625); M42C - Martensitic Stainless Steel (SS 431).

The manufacturing processes for FGMs generally include two steps: constructing the non-homogeneous structure, called "gradation", and transforming this structure into a bulk material, called "consolidation" [5]. However, using DLD, FGM production is a one-step process, diminishing production time and enlarging the material systems that can be combined.

The newest DLD machines can have up to four nozzles (or more) with inert gas protection, allowing different combinations of powders and minimizing the oxidation resulting from the elevated temperature used on metal processing. Laser plays an essential role in DLD, and its development in terms of power and efficiency has impacted the growth of metal AM technologies. However, besides the laser choice, several other parameters must be considered during DLD, namely: substrate, laser power, scanning speed, laser scanning pattern, laser beam diameter, hatch spacing, powder feed rate, powders composition, powder gradient variation, and preheating conditions $[4,6]$. The processing conditions selection ensuring a cladding without defects, bonded to the substrate, and with good material yield, is an essential and challenging task, as the various variables interact simultaneously, and some in opposite directions.

This technique can create near net shape parts from powder or wire, melting them into the desired geometry, ensuring excellent bonding and metallurgical properties [7-10]. Due to its geometric freedom, scalability, and adaptability to distinct scenarios, which other metals AM technologies cannot offer, DLD is often used to produce complex and custom parts and coating/repairing metal components $[6,11,12]$. Still, the great advantage of this technology is the possibility of processing several materials in the same operation, granted with several powder feeders. Therefore, this technology can be assumed to be a multi material/graded deposition method. Different powders can be used simultaneously, enabling the deposition of distinct materials in consecutive layers or combining different amounts of material in each layer.

Although DLD appears as a practical option for manufacturing Metallic FGMs, the inherent process complexity must be adequately addressed. Indeed, laser-deposited materials experience complicated thermal history: rapid solidification, high cooling rates, steep thermal gradients, and cyclic reheating (including localized remelting) and cooling. These phenomena produce nonequilibrium microstructures and significant structural variations from layer to layer and even within individual layers. Furthermore, the final microstructure and properties are intimately connected to the mentioned process parameters, which must be optimized for each specific case $[13,14]$.

In fact, producing FGMs requires knowledge of thermal and thermophysical properties of materials used: coefficient of thermal expansion (CTE), melting temperature, and thermal conductivity. The inequality in the heat flow is due to faster dissipation at the material interface that presents higher thermal conductivity, resulting in distortion and the possibility of lack of fusion in the other material, resulting from insufficient heat [14].

Thermal expansion differences and lattice misfits can induce high residual stresses in FGMs. In DLD, the extremely high thermal gradient enhances thermal stresses; these are related to the expansion of the melt pool and the contraction during rapid cooling [15]. These mismatches can arise between FGM first layers and substrate and through the FGM, causing cracks or interfacial delamination between the clad and the substrate or between the FGM layers. The distribution of these stresses is highly dependent on the substrate and the FGM materials and can be tensile on the substrate and compressive on the FGM or vice versa.

However, experimental and numerical results proved that distributing the composition gradient over a larger number of layers reduces the CTEs difference and lattice misalignment, thus minimizing the residual stress $[16,17]$. The thickness and composition of each layer are also critical factors in this effect [18]. In addition, processing conditions determine the magnitude of residual stresses, decisive in processes in which thermal energy is quickly transferred to the cold substrate, such as DLD.

Preheat treatment is a solution to reduce residual stresses in FGMs, by reducing the cooling rate of both the substrate and the cladding $[19,20]$, and attenuating the difference in thermal 
expansion coefficients between the several powders [21]. Thus, preheated substrates reduce the susceptibility to the formation of cracks, promoting better mechanical properties $[22,25]$.

The maintenance of preheating during the deposition process can increase its beneficial effect [26]. Preheating also inhibits the formation of fragile and harmful phases, such as eutectics and borides [27], thus reducing defects in FGMs.

Summing up, additive manufacturing, specifically DLD technology, brings the opportunity of an alternative and flexible process for FGMs production, enabling the production and repair of components with this type of functionality. The FGMs can be produced either in bulk, by deposition of several layers, or on the surface, depositing few layers.

\section{Funding}

This research was funded by FEDER through the Operational Programme for Competitiveness and Internationalization (COMPETE 2020), Projetos em Copromoção (project POCI-010247-FEDER-039848).

\section{Acknowledgement}

The authors are grateful to CEMUP (Centro de Materiais da Universidade do Porto) for expert assistance with SEM.

\section{Conflicts of Interest}

The authors declare no conflict of interest.

\section{Reference}

1. DT Sarathchandra, S Kanmani Subbu, N Venkaiah (2018) Functionally graded materials and processing techniques: An art of review. Mater Today Proc 5(10): 21328-21334.

2. GH Loh, E Pei, D Harrison, MD Monzón (2018) An overview of functionally graded additive manufacturing. Addit Manuf 23: 34-44.L Yan, Y Chen, F Liou (2020) Additive manufacturing of functionally graded metallic materials using laser metal deposition. Additive Manufacturing 31: 100901.

3. RM Mahamood (2018) Laser Metal Deposition of Metals and Alloys. Production \& Process Engineering.

4. B Kieback, A Neubrand, H Riedel (2003) Processing techniques for functionally graded materials. Materials Science and Engineering: A 362(12) $81-106$.

5. SM Thompson, L Bian, N Shamsaei, A Yadollahi (2015) An overview of Direct Laser Deposition for additive manufacturing; Part I: Transport phenomena, modeling and diagnostics. Additive Manufacturing 8: 3662.

6. A Saboori, G Piscopo, M Lai, A Salmi, S Biamino (2020) An investigation on the effect of deposition pattern on the microstructure, mechanical properties and residual stress of 316L produced by Directed Energy Deposition. Materials Science and Engineering: A 780: 139179.

7. W Paatsch (2016) Energy turnaround - a challenge for surface technology. Transactions of the IMF 94(5): 228-230.

8. Y Gao, D Xiong, C Wang, Y Chen (2009) Influences of laser powers on microstructure and properties of the coatings on the AZ91HP magnesium alloy. Acta Metallurgica Sinica 22(3): 167-173.
9. J Chen, SH Wang, L Xue (2012) On the development of microstructures and residual stresses during laser cladding and post-heat treatments. Journal of Materials Science 47(2): 779-792.

10. EP Cardozo, GR Pardal, S Ríos, S Ganguly, ASCM D’oliveira (2019) Additive techniques to refurbish Ni based components. Soldag Insp 24: 1-11.

11. Q Guo, S Chen, M Wei, J Liang, C Liu, et al. (2020) Formation and Elimination Mechanism of Lack of Fusion and Cracks in Direct Laser Deposition 24CrNiMoY Alloy Steel. Journal of Materials Engineering and Performance 29(10): 6439-6454.

12. L Costa, Vilar R (2009) Laser powder deposition. Rapid Prototyping Journal 15(4): 264-279.

13. A Reichardt (2017) Additive Manufacturing of Metal-based Functionally Graded Materials. Additive Manufacturing of Metal-based Functionally Graded Materials, UC Berkeley.

14. S Zhou, X Zeng, Q Hu, Y Huang (2008) Analysis of crack behavior for $\mathrm{Ni}$ based WC composite coatings by laser cladding and crack-free realization. Applied Surface Science 255(5): 1646-1653.

15. A Bhattacharyya, D Maurice (2019) Residual stresses in functionally graded thermal barrier coatings. Mechanics of Materials 129: 50-56.

16. XC Zhang, BS Xu, HD Wang, Y Jiang, YX Wu (2006) Modeling of thermal residual stresses in multilayer coatings with graded properties and compositions. Thin Solid Films 497(1-2): 223-231.

17. KZ Uddin, B Koohbor (2020) Gradient optimization of transversely graded Ti-TiB structures for enhanced fracture resistance. Int J Mech Sci 187: 105917.

18. G Bidron, A Doghri, T Malot, F Fournier-dit-Chabert, M Thomas, et al. (2020) Reduction of the hot cracking sensitivity of CM-247LC superalloy processed by laser cladding using induction preheating. J Mater Process Technol 277: 116461.

19. A Sadhu, Choudhary A, Sarkar S, Nair AM, Nayak P, et al. (2020) A study on the influence of substrate pre-heating on mitigation of cracks in direct metal laser deposition of NiCrSiBC- $60 \%$ WC ceramic coating on Inconel 718. Surf Coatings Technol 389: 125646.

20. R Ma, Z Liu, W Wang, G Xu, W Wang (2020) Microstructures and mechanical properties of Ti6Al4V-Ti48Al2Cr2Nb alloys fabricated by laser melting deposition of powder mixtures. Mater Charact 164: 110321.

21. C Ding, X Cui, J Jiao, P Zhu (2018) Effects of substrate preheating temperatures on the microstructure, properties, and residual stress of $12 \mathrm{CrNi} 2$ prepared by laser cladding deposition technique. Materials $11(12)$.

22. K Dai, XX Li, LL Shaw (2004) Comparisons between thermal modeling and experiments: Effects of substrate preheating. Rapid Prototyp J 10(1): 24-34.

23. B Zheng, Y Zhou, JE Smugeresky, JM Schoenung, EJ Lavernia (2008) Thermal behavior and microstructural evolution during laser deposition with laser-engineered net shaping: Part I. Numerical calculations. Metall Mater Trans A Phys Metall Mater Sci 39(9): 2228-2236.

24. AA Ferreira, R Darabi, JP Sousa, JM Cruz, AR Reis, et al. (2021) Optimization of direct laser deposition of a martensitic steel powder (Metco 42c) on 42 crmo4 steel. Metals 11(4): 1-18.

25. A Thiriet, C Schneider-Maunoury, P Laheurte, D Boisselier, L Weiss (2019) Multiscale study of different types of interface of a buffer material in powder-based directed energy deposition: Example of Ti6Al4V/ Ti6Al4V - Mo/Mo - Inconel 718. Additive Manufacturing 27: 118-130.

26. A Ramakrishnan, GP Dinda (2019) Direct laser metal deposition of Inconel 738. Materials Science and Engineering: A A740-741. 